\title{
Superfluid Filaments of Dipolar Bosons in Free Space
}

\author{
Fabio Cinti, ${ }^{1,2, *}$ Alberto Cappellaro, ${ }^{3, \dagger}$ Luca Salasnich, ${ }^{3,4,+}$ and Tommaso Macrì ${ }^{5,8}$ \\ ${ }^{1}$ National Institute for Theoretical Physics (NITheP), Stellenbosch 7600, South Africa \\ ${ }^{2}$ Institute of Theoretical Physics, Stellenbosch University, Stellenbosch 7600, South Africa \\ ${ }^{3}$ Dipartimento di Fisica e Astronomia Galileo Galilei and CNISM, Università di Padova, via Marzolo 8, 35131 Padova, Italy \\ ${ }^{4}$ CNR-INO, via Nello Carrara, 1-50019 Sesto Fiorentino, Italy \\ ${ }^{5}$ Departamento de Física Teórica e Experimental, Universidade Federal do Rio Grande do Norte, \\ and International Institute of Physics, 59078-970 Natal, Rio Grande do Norte, Brazil
}

(Received 29 October 2016; revised manuscript received 9 July 2017; published 20 November 2017)

\begin{abstract}
We systematically investigate the zero temperature phase diagram of bosons interacting via dipolar interactions in three dimensions in free space via path integral Monte Carlo simulations with a few hundreds of particles and periodic boundary conditions based on the worm algorithm. Upon increasing the strength of the dipolar interaction and at sufficiently high densities we find a wide region where filaments are stabilized along the direction of the external field. Most interestingly by computing the superfluid fraction we conclude that the superfluidity is anisotropic and is greatly suppressed along the orthogonal plane. Finally, we perform simulations at finite temperature confirming the stability of the filaments against thermal fluctuations and provide an estimate of the superfluid fraction in the weak coupling limit in the framework of the Landau two-fluid model.
\end{abstract}

DOI: 10.1103/PhysRevLett.119.215302

Superfluidity is an amazing phenomenon of quantum mechanical origin that manifests itself macroscopically as frictionless flow and a lack of response to rotation for a small enough angular velocity $[1,2]$. Several experimental platforms have been used to investigate quantum matter in the superfluid regime [3]. Among them, ultracold gases realize a very clean and controllable many-body playground that permits the observation of quantum properties with unprecedented precision [4]. In these systems superfluidity has been observed both with bosonic as well as with fermionic atoms [5-8]. The superfluid fraction, the ratio of the superfluid density to the total density of the system, has been recently measured in a two-component Fermi gas interacting via strong contact potentials $[7,9,10]$.

An even richer phenomenology appears when longrange interactions are present. The nonlocal character of the interparticle potential may induce instabilities of the density that lead to a spontaneous breaking of translational symmetry. A primary example is the long sought supersolid state, where superfluidity is accompanied by a crystalline order [11-14]. Recent ground-breaking experiments with dipolar condensates demonstrated the existence of dense bosonic droplets in trapped configurations [15-18] and in free space [19]. Beyond mean-field effects [20-22] and three-body interactions [23] have been invoked as the main mechanisms responsible for the stability of these clusters. Large scale simulations based on a nonlocal nonlinear Schrödinger equation have shown very good agreement with the density distribution and the excitation spectra observed in the laboratory. The latest experiments paved the way for the search for the phase coherence of droplets, demonstrating an interference pattern via the expansion dynamics of the condensate. The presence of fringes showed that each droplet is individually phase coherent and thus superfluid, leaving yet unresolved the question of the global phase coherence of the system [16].

In this Letter we report path-integral Monte Carlo (PIMC) results for the low temperature properties of a finite size system of dipolar bosons in three-dimensional free space. Recent works showed with similar methods the existence of a window in parameter space leading to stable self-bound configurations of a single [24] or several droplets in a regular arrangement in trapped configurations [25]. Yet, no work has investigated the superfluid character of these structures. Here, we address the issue of superfluidity for a wide range of the strength of the dipolar interaction and density as well as their stability against finite temperature fluctuations. We carry out large scale simulations, based on a continuous-space worm algorithm that allows for an efficient and reliable determination of the superfluid density [26,27]. We observe an anisotropic character of the superfluid fraction in the inhomogeneous regime of the filaments. Our results are consistent with the absence of supersolidity in these systems.

The Hamiltonian of an ensemble of $N$ interacting identical bosons is

$$
\hat{H}=-\sum_{i=1}^{N} \frac{\hbar^{2}}{2 m} \nabla_{i}^{2}+\sum_{i<j}^{N} V\left(\mathbf{r}_{i}-\mathbf{r}_{j}\right),
$$

where $m$ is the particle mass, and $\mathbf{r}_{i}$ is the position of the $i$ th dipole. We model the interparticle potential $V(\mathbf{r})$ by a shortrange hard core with cutoff $r_{0}$ and an anisotropic longrange dipolar potential: 


$$
V(\mathbf{r})= \begin{cases}\frac{C_{d d} \frac{1-3 \cos ^{2} \theta}{4 \pi},}{r^{3}}, & \text { if } r \geq r_{0}, \\ \infty, & \text { if } r<r_{0}\end{cases}
$$

where $C_{d d} / 4 \pi$ is the coupling constant and the angle $\theta$ denotes the angle between the vector $\mathbf{r}$ and the $z$ axis. Here, the units of length and energy are $r_{0}$ and $\hbar^{2} / m r_{0}^{2}$, respectively. Therefore, the zero-temperature physics is controlled by the dimensionless interaction strength $U=$ $m C_{d d} / 4 \pi \hbar^{2} r_{0}$ and the dimensionless density $n r_{0}^{3}$ only [28]. Equation (1) applies both to vertically aligned dipoles interacting via magnetic or electric dipole moments [31]. The hard core with radius $r_{0}$ removes the unphysical $1 / r^{3}$ attraction of dipoles in head-to-tail configurations at small distances. Finite dipolar potentials affect the scattering length $a_{s}$ associated with the contact potential into a nontrivial relation of $C_{d d}[29,30,32]$. This turns out to be crucial to account for the stability properties of a dipolar gas in generic (beyond) mean-field approaches. For example, a standard Bogoliubov calculation determines the stability of a homogeneous condensate when $\epsilon_{d} \equiv a_{d} / a_{s}<$ 1 is satisfied, where $a_{d}=\left(C_{d d} m / 12 \pi \hbar^{2}\right)$ is the dipolar length associated with Eq. (2) $[31,33]$.

We determine the equilibrium properties of Eq. (1) in a wide range of scaled average density $n r_{0}^{3}$ (different from the experimentally measured peak density) and interaction strength $U$. We work with $N$ atoms in a cubic box of linear dimension $L$ and periodic boundary conditions. At fixed density $n=N / L^{3}$, with $N=100-400$, we verified that the phase diagram does not change. Thus, our finite-size results give a reasonable estimate of the thermodynamic limit. [34]. From these simulations we obtain, for example, density profiles, and radial correlation functions, as well as the superfluid fraction [35] at finite temperatures. The phases were obtained by extrapolating to the limit of zero temperature, lowering the temperature until observables, such as the total energy, superfluid fraction $f_{s}$, and radial correlations, did not change on further decrease of $T$. These observables were then analyzed to construct the phase diagram in Fig. 1. For small $U$ the system is in a superfluid phase (SF) with unitary $f_{s}$. For low densities $n r_{0}^{3} \lesssim 10^{-3}$ the SF extends up to $U \approx 2.1$, which agrees with the mean-field phase boundary predicted by standard Bogoliubov analysis $\left(\epsilon_{d}=1\right)$ for the dipolar potential and a contact interaction [36]. Increasing the interaction strength the system enters into a cluster phase (CP) with vanishing superfluidity and characterized by droplet structures with few particles. For higher densities $n r_{0}^{3}>6 \times 10^{-4}$, crossing the SF phase boundary, we encounter a phase characterized by elongated filaments (FP) with an anisotropic superfluid fraction. We notice that this FP extends from a small positive induced contact potential $\left(\epsilon_{d} \gtrsim 1\right)$, which corresponds to the experimentally relevant regime of Refs. [15,36-42], to the strongly coupled limit of large dipolar interactions and large densities. For

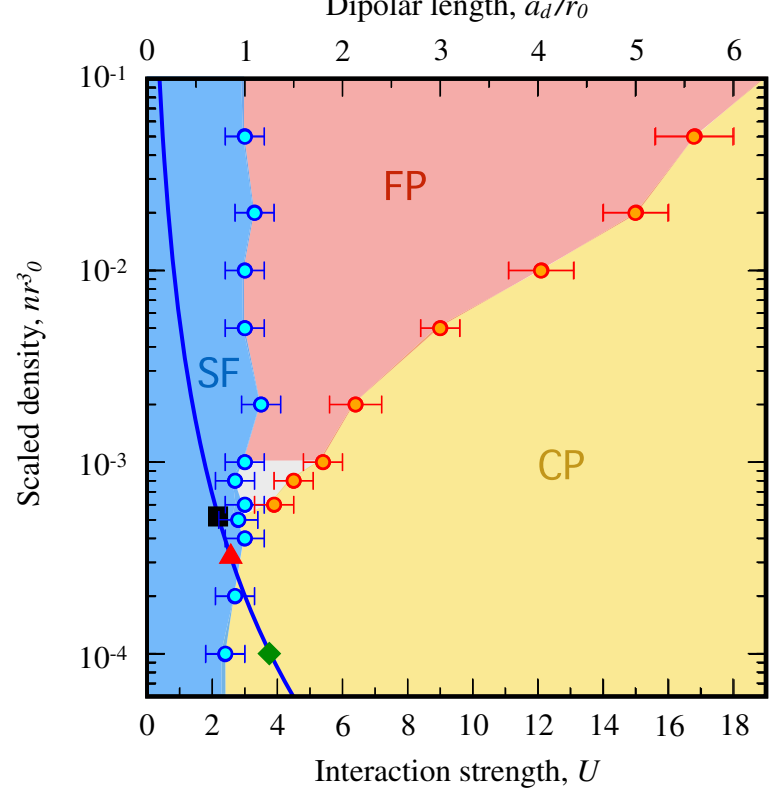

FIG. 1. Zero-temperature phases of dipolar bosons in three dimensions in free space: superfluid (SF), superfluid filaments (FP), and cluster phases (CP) for varying dimensionless interaction strength $U$ (or equivalently dipolar length $a_{d} / r_{0}$ ) and scaled density $n r_{0}^{3}$. For $a_{d} / r_{0} \lesssim 0.9$ the blue SF region corresponds to a uniform superfluid, which agrees with the mean-field prediction that predicts stability for $a_{d} / a_{s}<1$. FPs are encountered for high densities $n r_{0}^{3} \gtrsim 5 \times 10^{-4}$ and for $a_{d} / r_{0} \gtrsim 1$. For small densities $n r_{0}^{3} \lesssim 5 \times 10^{-4}$ the system breaks up into tiny clusters. Phase boundaries in the gray region are not resolved. The blue line corresponds to $n=5 \times 10^{20} \mathrm{~m}^{-3}$ and $a_{d}=130 a_{0}$. The black [red] dots are the experimentally measured background scattering lengths $a_{s}=122 a_{0}\left({ }^{162} \mathrm{Dy}\right)\left[a_{s}=92 a_{0}\left({ }^{164} \mathrm{Dy}\right)\right]$. The green point at $a_{s}=29 a_{0}$ [36].

intermediate densities $6 \times 10^{-4} \lesssim n r_{0}^{3} \lesssim 1 \times 10^{-3}$ the phase boundaries are not resolved.

Representative PIMC configurations with 100 particles and 500 imaginary-time slices $\left(n r_{0}^{3}=10^{-2}\right)$ of the density distribution are shown in Fig. 2. In the SF [Fig. 2(a)] particles delocalize into a phase with a uniform density displaying flat radial distribution functions $g(r)$, typical of a fluid phase of particles interacting via hard-core potentials. In Fig. 2(b) a typical configuration of the FP is illustrated where filaments elongate vertically. Finally, in the CP several small clusters form, slightly elongated along the vertical direction, signaling a fragmentation of the filaments for large interactions. In the nonhomogeneous FP and CP we analyze $g(r)$ along two orthogonal directions. The function $g_{\|}(r)$ (red line in Fig. 2) is computed along the vertical axis parallel to the direction of the filaments. In the FP $g_{\|}(r)$ has a liquidlike profile with a peak $r \gtrsim r_{0}$ as a consequence of the attractive part of the dipolar interaction and the large density along the $z$ axis. In the CP the first peak of $g_{\|}(r)$ is higher and the function oscillates several times before vanishing for large distances as a consequence 

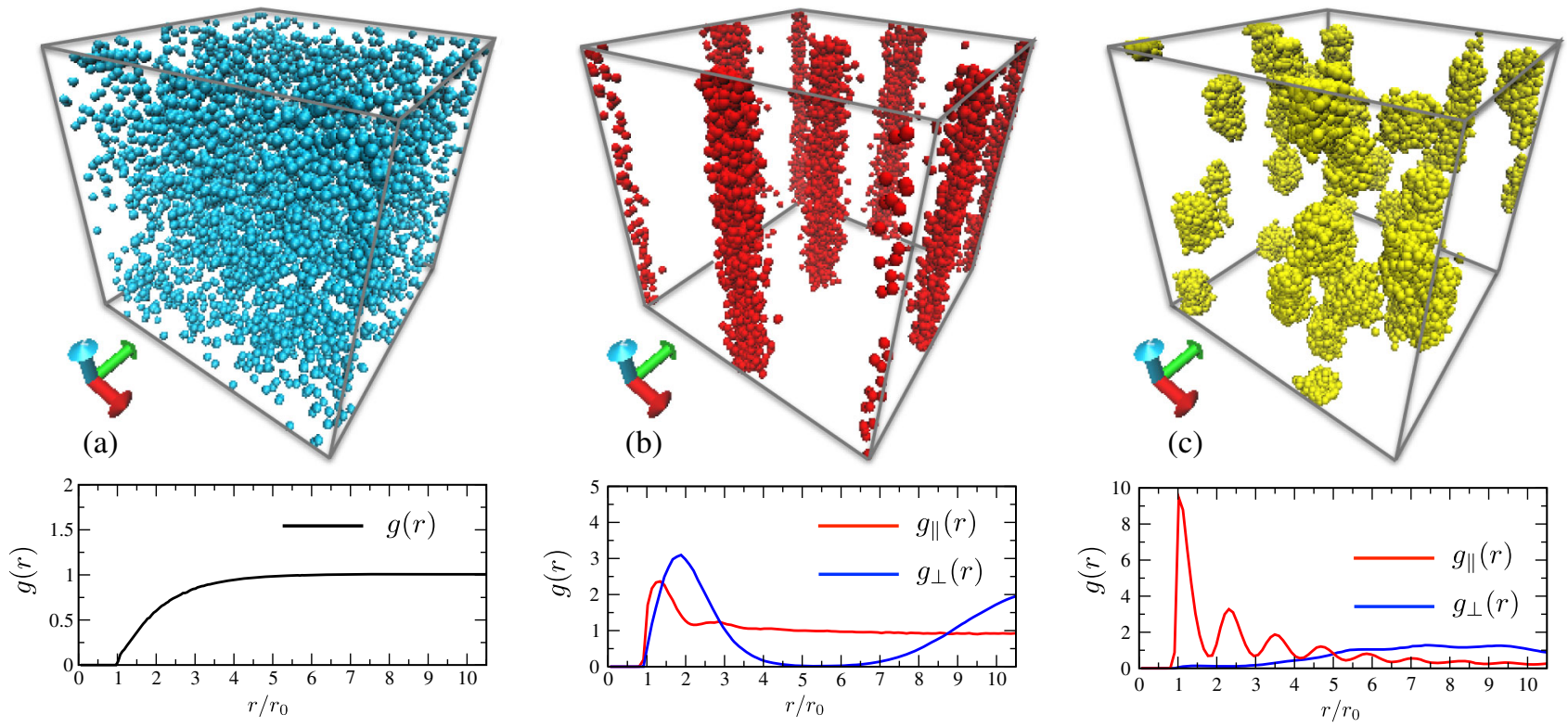

FIG. 2. Characterization of the phases at $n r_{0}^{3}=10^{-2}$. Upper panels. PIMC density distribution at different strengths of the dipolar interaction: (a) the SF at $a_{d}=0.6 r_{0}$, (b) the superfluid FP at $a_{d}=2.6 r_{0}$ and (c) the $\mathrm{CP}$ at $a_{d}=6.0 r_{0}$. Lower panels. (a) radial correlation functions $g(r)$ in the SF. (b),(c) Radial correlation functions $g_{\|}(r)$ along the vertical direction (red) and $g_{\perp}(r)$ along the orthogonal plane (blue). $g_{\|}(r)$ has a fluidlike profile in the FP. In the CP $g_{\|}(r)$ shows a high peak due to strong attractive interactions appears at distances $r \gtrsim r_{0} . g_{\perp}(r)$ in the FP shows a strong suppression in between two filaments whereas in the CP it flattens at intermediate distances. Simulations are done with 100 particles and 500 slices. Correlation functions are averaged over 100 configurations.

of the finite size of the clusters. The correlation $g_{\perp}(r)$ (blue line) is evaluated along the horizontal plane, perpendicular to the direction of the filaments. In the FP, it is strongly suppressed in the region between two filaments. In the CP $g_{\perp}(r)$ flattens at intermediate distances displaying an irregular arrangement of the small dipolar droplets along the horizontal plane.

To fully characterize the genuine quantum properties of the FP we compute the superfluid fraction $f_{s}^{(i)}$ as a function of the interaction strength and temperature along three orthogonal directions $i=x, \quad y, \quad z$, yielding $f_{s}^{(i)}=$ $\left(m k_{B} T / \hbar^{2} n\right)\left\langle w_{i}^{2}\right\rangle$, where $\langle\cdots\rangle$ stands for the thermal average of the winding number estimator $w_{i}[43,44]$. Figure 3 depicts the superfluid fraction $f_{s}^{(i)}$ calculated varying the dipolar length $a_{d}$ for $n r_{0}^{3}=10^{-2}$. In the SF the superfluid density is uniform and unitary $\left(f_{s}^{(i)}=1\right)$. Crossing the SF-FP phase boundary $f_{s}^{(i)}$ displays a strong anisotropy. The superfluid fraction is unitary along the vertical direction and it is greatly suppressed along the orthogonal $x y$ plane, showing only a marginal finite-size effect contribution to $w_{x}$ and $w_{y}$, respectively. This result suggests that each filament is phase coherent, but globally the system is not. This suppression of superfluidity along two directions resembles the formation of a sliding phase in weakly coupled superfluid layers in the thermodynamic limit [45-48]. Finally, entering in the $\mathrm{CP} f_{s}^{(i)}=0$ uniformly as expected from the fragmentation of the filaments in the configurations. The lower panel of Fig. 3 reports the relative frequency of exchange cycles involving a particle number $1 \leq L \leq N$ [49]. In the $\mathrm{SF}$ we observe long permutation cycles $(\sim N)$. In the FP $(\mathrm{CP})$ cycles reduce to few tens of (few) particles in support of the previous observation that superfluidity originates within each filament (locally within each cluster).

Finally, we investigate the stability of superfluid filaments at finite temperatures. In Fig. 4 we show the superfluid fraction for a system of $N=100$ particles at density $n r_{0}^{3}=$ $10^{-2}$ as a function of $T / T_{0}, T_{0}$ being the critical temperature of an ideal Bose gas $k_{B} T_{0}=\left[2 \pi / \zeta(3 / 2)^{2 / 3}\right]\left(n r_{0}^{3}\right)^{2 / 3}$ [50]. In the SF we compare PIMC results with an analytical calculation within the Landau two-fluid model [52]

$f_{s}^{(z)}=1-\frac{\beta \hbar^{2}}{4 \pi^{2} m} \int_{0}^{\infty} d q \int_{0}^{\pi} d \theta_{q} q^{4} \frac{\sin \theta_{q} \cos ^{2} \theta_{q} e^{\beta E_{q}}}{\left(e^{\beta E_{q}}-1\right)^{2}}$,

where $E_{\mathbf{q}}=\sqrt{\left(\hbar^{2} q^{2} / 2 m\right)\left[\left(\hbar^{2} q^{2} / 2 m\right)+2\left(\mu / g_{0}\right) \tilde{V}_{\mathrm{ps}}(\mathbf{q})\right]}$ is the Bogoliubov spectrum. The function $\tilde{V}_{\mathrm{ps}}(\mathbf{q})=g_{0}\left[1-\epsilon_{d}+\right.$ $\left.3 \epsilon_{d} \cos ^{2}\left(\theta_{q}\right)\right]$ is the Fourier transform of the pseudopotential $V_{\mathrm{ps}}(\mathbf{r})=g_{0} \delta(\mathbf{r})+\left(C_{d d} / 4 \pi\right)\left(1-3 \cos ^{2} \theta / r^{3}\right)$, widely used as a regularized form of the interparticle potential (2), with $g_{0}=4 \pi \hbar^{2} a_{s} / m \quad[31,33,53]$. The chemical potential $\mu$ includes the beyond mean-field contribution of the dipolar interaction: 

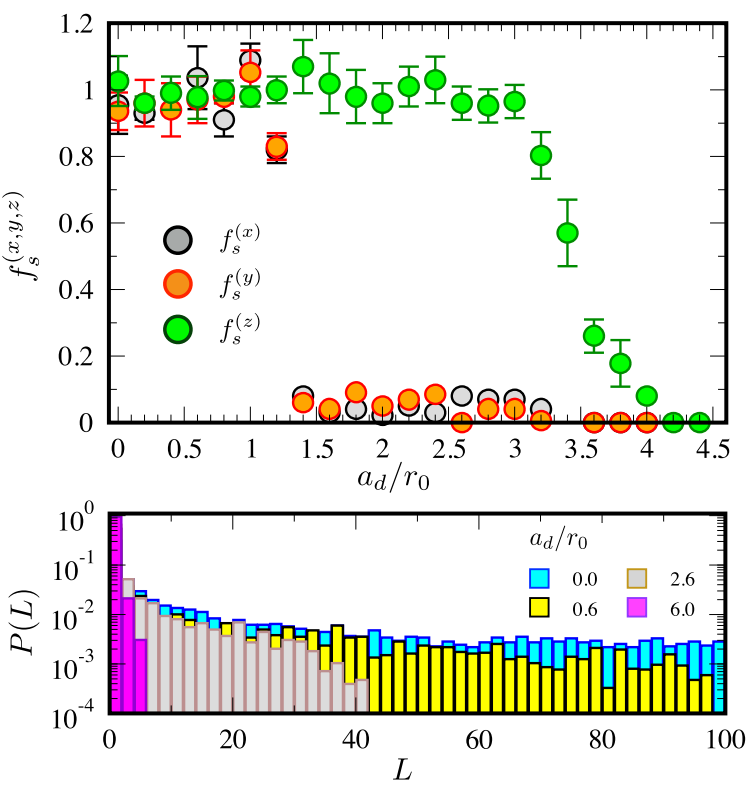

FIG. 3. Superfluid fraction $f_{s}$ across the transition from superfluid to filament at high densities. Upper panel. $f_{s}$ as a function of the dipolar length along the vertical direction and the orthogonal direction for $n r_{0}^{3}=10^{-2}$. Dipoles and filaments are aligned along the $z$ axis. In the SF the superfluid fraction converges to $f_{s}=1$ isotropically. In the filaments we observe $f_{s}=1$ along the vertical axis and vanishing on the orthogonal plane. Error bars are statistical uncertainties. Lower panel. Relative frequency of permutation cycles of length $L$ again for $n r_{0}^{3}=10^{-2}$ and $a_{d}=0$, $0.6,2.8,6.0 r_{0}$ in the SF (blue and yellow), the FP (gray), and the $\mathrm{CP}$ (purple).

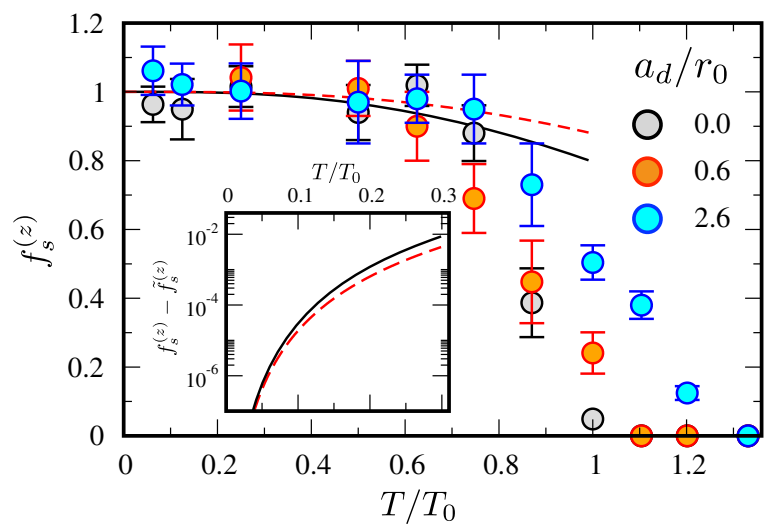

FIG. 4. Superfluid fraction $f_{s}^{(z)}$ as a function of the scaled temperature $T / T_{0}$ at $n r_{0}^{3}=10^{-2}$ for three values of the dipolar lengths: (a) the SF with $a_{d}=0$ (hard-core bosons), (b) the SF with $a_{d}=0.6 r_{0}$, (c) the FP with $a_{d}=2.6 r_{0}$. The lines refer to the analytical prediction of the temperature dependence of the superfluid fraction at $n r_{0}^{3}=10^{-2}$ from Eq. (3) at $a_{d}=0$ (black continuous line) and $a_{d}=0.6 r_{0}$ (red dashed line). Inset: low temperature limit of $f_{s}^{(z)}$ from Eq. (3) and Eq. (5) in the SF at $a_{d}=0$ and $a_{d}=0.6 r_{0}$.

$$
\mu=g_{0} n\left(1+\frac{32}{3 \sqrt{\pi}} \sqrt{n a_{s}^{3}} Q_{5 / 2}\left(\epsilon_{d}\right)\right),
$$

where $Q_{\alpha}(x)=\int_{0}^{1} d t\left(1-x+3 x t^{2}\right)^{\alpha}$ [54]. The plot of the superfluid fraction along the vertical axis from Eq. (3) is shown in Fig. 4 for $a_{d}=0$ (black line) and $a_{d}=0.6 r_{0}$ (red dashed line). In the low temperature regime we can linearize $E_{\mathbf{q}}$ to determine an approximate analytical formula for the superfluid fraction

$$
\tilde{f}_{s}^{(z)}=1-\frac{2 \pi^{2}}{45} \frac{1}{\left(1-\epsilon_{d}\right)\left(1+2 \epsilon_{d}\right)^{3 / 2}}\left(\frac{m}{\hbar^{2}}\right)^{3 / 2} \frac{\left(k_{B} T\right)^{4}}{n \mu^{5 / 2}}
$$

that works well up to $T \lesssim 0.3 T_{0}$. In the inset of Fig. 4 we show the difference between the superfluid fractions of Eq. (3) and the analytical result (5) for the same values of $a_{d}$ as in the main figure. For a larger dipolar length $a_{d}=2.6 r_{0}$, in the FP, the superfluid fraction along the vertical axis is finite within a large window of temperatures. We conclude then that filaments are stable against finite temperature fluctuations and we clearly see that anisotropic superfluidity is finite up to temperatures $T \sim 0.8 T_{0}$. We also verified that the orthogonal components of the superfluid fraction are vanishing in this regime.

Our results then suggest that the observed interference pattern [15] is a consequence of local phase coherence (within each droplet) and not of a global one. As discussed above the FP is characterized by an anisotropic superfluid density that, interestingly, might be measured via the second sound along the orthogonal directions as done with strongly interacting Fermi gases [5-8].

In this Letter we studied the many-body phases of an ensemble of bosons interacting via dipole-dipole interactions in three-dimensional free space and investigated the superfluid behavior of dipolar filaments. We spanned a wide range of the parameter space confirming the existence of an extended phase of filaments with unitary superfluidity along the vertical direction and vanishing otherwise. Our results therefore theoretically support recent experimental findings about the stability of droplets in free space [19] and the presence of local phase coherence [15] giving rise to interference fringes but excluding global phase coherence of the filaments [36] and therefore a possible supersolid phase. Finally, we confirmed that the filaments are stable at finite temperature. More refined investigations are needed to determine accurately the melting transition of the filaments into a fluid phase.

We thank R. N. Bisset, M. Boninsegni, S. Giorgini, G. Gori, A. Pelster, T. Pohl, G. Shlyapnikov, F. Toigo, and M. Troyer for useful discussions and I. Ferrier-Barbut for helpful correspondence. T. M. acknowledges Conselho Nacional de Desenvolvimento Científico e Tecnológico for support through Bolsa de produtividade em Pesquisa $\mathrm{n}$. 311079/2015-6 and thanks NITheP for the hospitality where part of the work was done. F. C. acknowledges the hospitality of the MPI-PKS in Dresden where part of the work was carried out. 
*inti@sun.ac.za

†cappellaro@pd.infn.it

*luca.salasnich@unipd.it

§macri@fisica.ufrn.br

[1] L. Pitaevskii and S. Stringari, Bose-Einstein Condensation (Oxford University Press, New York, 2003).

[2] C. Pethick and H. Smith, Bose-Einstein Condensation in Dilute Gases (Cambridge University Press, Cambridge, England, 2002).

[3] A. J. Leggett, Quantum Liquids Bose Condensation and Cooper Pairing in Condensed-Matter Systems (Oxford University Press, New York, 2006).

[4] I. Bloch, J. Dalibard, and W. Zwerger, Rev. Mod. Phys. 80, 885 (2008).

[5] L. Verney, L. Pitaevskii, and S. Stringari, Europhys. Lett. 111, 40005 (2015).

[6] M. K. Tey, L. A. Sidorenkov, E. R. Sanchez Guajardo, R. Grimm, M. J. H. Ku, M. W. Zwierlein, Y.-H. Hou, L. Pitaevskii, and S. Stringari, Phys. Rev. Lett. 110, 055303 (2013).

[7] L. P. Pitaevskii and S. Stringari, arXiv:1510.01306.

[8] Y.-H. Hou, L. P. Pitaevskii, and S. Stringari, Phys. Rev. A 88, 043630 (2013).

[9] L. Salasnich, Phys. Rev. A 82, 063619 (2010).

[10] L. A. Sidorenkov, M. K. Tey, R. Grimm, Y.-H. Hou, L. Pitaevskii, and S. Stringari, Nature (London) 498, 78 (2013).

[11] M. Boninsegni and N. V. Prokofev, Rev. Mod. Phys. 84, 759 (2012).

[12] F. Cinti, T. Macrì, W. Lechner, G. Pupillo, and T. Pohl, Nat. Commun. 5, 3235 (2014); F. Cinti, P. Jain, M. Boninsegni, A. Micheli, P. Zoller, and G. Pupillo, Phys. Rev. Lett. 105, 135301 (2010); S. Saccani, S. Moroni, and M. Boninsegni, Phys. Rev. B 83, 092506 (2011); M. Boninsegni, J. Low Temp. Phys. 168, 137 (2012); S. Saccani, S. Moroni, and M. Boninsegni, Phys. Rev. Lett. 108, 175301 (2012); T. Macrì, F. Maucher, F. Cinti, and T. Pohl, Phys. Rev. A 87, 061602 (R) (2013); T. Macrì, S. Saccani, and F. Cinti, J. Low Temp. Phys. 177, 59 (2014).

[13] J. Leonard, A. Morales, P. Zupancic, T. Esslinger, and T. Donner, Nature (London) 543, 87(2017).

[14] J. Li, J. Lee, W. Huang, S. Burchesky, B. Shteynas, F. C. Top, A. O. Jamison, and W. Ketterle, Nature (London) 543, 91 (2017).

[15] H. Kadau, M. Schmitt, M. Wenzel, C. Wink, T. Maier, I. Ferrier-Barbut, and T. Pfau, Nature (London) 530, 194 (2016).

[16] I. Ferrier-Barbut, H. Kadau, M. Schmitt, M. Wenzel, and T. Pfau, Phys. Rev. Lett. 116, 215301 (2016).

[17] L. Chomaz, S. Baier, D. Petter, M. J. Mark, F. Wachtler, L. Santos, and F. Ferlaino, Phys. Rev. X 6, 041039 (2016).

[18] I. Ferrier-Barbut, M. Schmitt, M. Wenzel, H. Kadau, and T. Pfau, J. Phys. B 49, 214004 (2016).

[19] M. Schmitt, M. Wenzel, F. Bottcher, I. Ferrier-Barbut, and T. Pfau, Nature (London) 539, 259 (2016).

[20] F. Wachtler and L. Santos, Phys. Rev. A 93, 061603 (2016).

[21] R. N. Bisset, R. M. Wilson, D. Baillie, and P. B. Blakie, Phys. Rev. A 94, 033619 (2016).
[22] D. Baillie, R. M. Wilson, R. N. Bisset, and P. B. Blakie, Phys. Rev. A 94, 021602 (2016).

[23] R. N. Bisset and P. B. Blakie, Phys. Rev. A 92, 061603 (2015).

[24] H. Saito, J. Phys. Soc. Jpn. 85, 053001 (2016).

[25] A. Macia, J. Sanchez-Baena, J. Boronat, and F. Mazzanti, Phys. Rev. Lett. 117, 205301 (2016).F. Cinti and M. Boninsegni, Phys. Rev. A 96, 013627 (2017).

[26] M. Boninsegni, N. Prokof'ev, and B. Svistunov, Phys. Rev. Lett. 96, 070601 (2006).

[27] M. Boninsegni, N. V. Prokof'ev, and B. V. Svistunov, Phys. Rev. E 74, 036701 (2006).

[28] The scaled density $n r_{0}^{3}$ coincides with the usual definition of the gas parameter $n a_{s}^{3}$ in the limit of vanishing dipolar interaction, that is, $C_{d d} \rightarrow 0$. See also below and Refs. [29,30]

[29] D. C. E. Bortolotti, S. Ronen, J. L. Bohn, and D. Blume, Phys. Rev. Lett. 97, 160402 (2006).

[30] S. Ronen, D. C. E. Bortolotti, D. Blume, and J. L. Bohn, Phys. Rev. A 74, 033611 (2006).

[31] T. Lahaye, C. Menotti, L. Santos, M. Lewenstein, and T. Pfau, Rep. Prog. Phys. 72, 126401 (2009).

[32] R. Oldziejewski and K. Jachymski, Phys. Rev. A 94, 063638 (2016).

[33] M. A. Baranov, M. Dalmonte, G. Pupillo, and P. Zoller, Chem. Rev. 112, 5012 (2012).

[34] A strong decay of the dipolar potential with distance precludes relevant effects of self-interaction, although neighbor images contribute.

[35] B. Svistunov, E. Babaev, and N. Prokofe'ev, Superfluid States of Matter (Taylor \& Francis, London, 2015).

[36] See Supplemental Material at http://link.aps.org/ supplemental/10.1103/PhysRevLett.119.215302 for a through comparison of our results with experiments with Dy and the discussion of the weak coupling limit.

[37] S. Yi and L. You, Phys. Rev. A 61, 041604(R) (2000).

[38] S. Yi and L. You, Phys. Rev. A 63, 053607 (2001).

[39] Y. Tang, A. Sykes, N. Q. Burdick, J. L. Bohn, and B. L. Lev, Phys. Rev. A 92, 022703 (2015).

[40] T. Maier, I. Ferrier-Barbut, H. Kadau, M. Schmitt, M. Wenzel, C. Wink, T. Pfau, K. Jachymski, and P. S. Julienne, Phys. Rev. A 92, 060702(R) (2015).

[41] N. Q Burdick, A. Sykes, Y. Tang, and B. L Lev, New J. Phys. 18, 113004 (2016).

[42] P. M. Chaikin and T. C. Lubensky, Principles of Condensed Matter (Cambridge University Press, Cambridge, England, 1995).

[43] E. L. Pollock and D. M. Ceperley, Phys. Rev. B 36, 8343 (1987).

[44] D. M. Ceperley, Rev. Mod. Phys. 67, 279 (1995).

[45] C. S. O'Hern, T. C. Lubensky, and J. Toner, Phys. Rev. Lett. 83, 2745 (1999).

[46] D. Pekker, G. Refael, and E. Demler, Phys. Rev. Lett. 105, 085302 (2010).

[47] N. Laflorencie, Europhys. Lett. 99, 66001 (2012).

[48] S. Vayl, A. B. Kuklov, and V. Oganesyan, Phys. Rev. B 95, 094101 (2017).

[49] P. Jain, F. Cinti, and M. Boninsegni, Phys. Rev. B 84, 014534 (2011). 
[50] An extensive calculation of the critical temperature with dipolar interactions is not reported here. Indeed, it can be done along the lines of Ref. [51] and it is the focus of a separate investigation.

[51] S. Pilati, S. Giorgini, and N. Prokof'ev, Phys. Rev. Lett. 100, 140405 (2008).
[52] M. Ghabour and A. Pelster, Phys. Rev. A 90, 063636 (2014).

[53] M. A. Baranov, Phys. Rep. 464, 71 (2008).

[54] A. R. P. Lima and A. Pelster, Phys. Rev. A 84, 041604 (2011). 